\title{
DIE TEXTSORTE "FERNSEHNACHRICHTEN" - KONTRASTIV BETRACHTET
}

Die Kenntnisse und Erfahrungen des einzelnen reichen heutzutage immer weniger hin, um die auf ihn einstürzende Flut zusammenhangloser Daten zu verarbeiten, so daß einmal mit der sich ständig ausweitenden Informationsdichte die Wahrscheinlichkeit geringer wird, daß der einzelne die für ihn wichtige Information erreicht. Und weiterhin wird durch die zunehmend der Öffentlichkeit entzogenen Entscheidungsinstanzen und Übermittlungswege die Einsichtmöglichkeit in den Zweck, in das erkenntnisleitende Interesse einer Information immer undurchsichtiger, so daß die Information nicht mehr als Grundlage rationaler Entscheidung, bzw. Handlung dienen kann.

Seit das Fernsehen in den 50er Jahren in Westeuropa und dann in den 60er Jahren im östlichen Mitteleuropa seinen regelmäßigen Programmdienst aufnahm, wurde die Mediendiskussion ständig durch die sich immer neu zu stellende Frage nach den Wirkungen und Möglichkeiten des Fernsehens belebt.

Der vorliegende Beitrag stellt einen Versuch dar, einerseits interkulturelle Einflüsse in der Gestaltung der Textsorte Fersehnachrichten bzw. Tagesschau zu beschreiben und andererseits spezifische sozio-kulturelle Merkmale festzustellen. Die Nachrichtensendungen kann man als Textsorte definieren, wenn im Bereich des Sprachlichen die Ebene des Textes zugleich als die Ebene der Redeakte bzw. der Gefüge von Redeakten bestimmt wird, die von einem bestimmten Sprecher in einer bestimmten Situation realisiert werden. Textsorten sind nicht nur als Medium für die Verständnisbildung bei Formulierern zu deuten. Aus der Sicht des Rezipienten haben sie den Status von Angeboten, sich auf der Basis des vorgegebenen Textes selber ein Verständnis zu bilden.

Die Nachrichtensendungen stellen eine Form der interpersonellen Kommunikation dar; der Kommunikationsprozeß ist indirekt und einseitig. Hinsichtlich der Gerichtetheit der kommunikativen Tätigkeit an die Adressaten handelt es sich bei den Nachrichtensendungen um eine öffentlichkeitsbestimmte Kommunikation. Für den Redakteur sind die Rezipienten zwar ein disperses Publikum, aber die Grundprinzipien der Informationsübermittlung sind trotz der Heterogenität der Empfänger durchführbar. Eines dieser für unseren Kulturkreis charakteristischen Grundprinzipien ist das Prinzip des objektiven Berichtens. Auf diesem Prinzip beruht auch der informative Wert der Nachrichten, denn ihrem öffentlichen Auftrag gemäß sind die Fernsehanstalten 
verpflichtet, ihren Beitrag zur politischen Meinungsbildung zu leisten und zwar so, daß sie streng zwischen Nachricht und Meinung unterscheiden. Politische Informationen sollten fürs Individuum kognitive und normative Funktionen erfüllen, obwohl sie sich quantitativ meistens ins Unübersehbare ausweiten - die Tagesschau bietet eine mit Formen und Inhalten überfrachtete, hektische Viertelstunde, in der pausenlos Nachricht an Nachricht gereiht und damit der gedankliche Nachvollzug nahezu völlig ausgeschaltet wird.

Gegenstand unserer Analyse waren die Sendungen Dnevnik 1 im kroatischen Fernsehen (täglich um 19.30 Uhr) sowie die ZDF-Sendung heute (täglich um $19 \mathrm{Uhr}$ ) und die Ö2-Sendung Zeit im Bild 2 (täglich um $22 \mathrm{Uhr}$ ). Diese Sendungen wurden zunächst unter dem Aspekt der Strukturierung der Nachrichtenblocks analysiert. Sowohl die Nachrichtensendungen im deutschen wie auch im österreichischen und im kroatischen Fernsehen werden nach dem gleichen Grundschema strukturiert: Nachrichten aus dem In-und Ausland, Sport, Wetterbericht.

Eine immer bestehende Gefahr in der Informationsübermittlung ist die Gefahr der Manipulation. Diese Gefahr besteht nicht nur auf der Ebene der sprachlichen Formulierung einer Nachricht; indirekt kann man auch durch die Übermittlung einer Nachricht, durch ihre Positionierung sowie durch die Zeitdauer der Präsentation den Rezipienten beeinflussen. Dabei ist Informieren mit Wertung engstens verbunden, denn die Struktur von Massenkommunikationsprozessen ist dadurch gekennzeichnet, daß Sprecher- und Hörerrollen von vornherein als fest und auf verschiedene Personengruppen bezogen definiert werden. Im Falle der Nachrichtensendungen gibt es nur den Redakteur bzw. Nachrichtensprecher und ihnen jeweils gegenüber die Menge der Zuschauer, die in bezug auf ihre Zuschauerrolle, d.h. in bezug auf ihr Interesse zu rezipieren, als Gruppe definiert werden kann.

Die Informationsübermittlung in der Textsorte Nachrichtensendung wurde anschließend unter den folgenden zwei Aspekten analysiert: Strukturierung der Sendung und Sprach- bzw. Sprechverhalten des Redakteurs. Die Grundstruktur ist in allen drei Nachrichtensendungen gleich. Am Anfang werden zusammenfassend die wichtigsten Ereignisse des Tages angeführt. Die Struktur dieser zusammenfassend angeführten wichtigsten Nachrichten ist mit der Struktur der Schlagzeilen in Zeitungsartikel vergleichbar - es handelt sich nämlich um knappe, elliptische und meist substantivische Konstruktionen: Neues Indiz, Eingeständnis, Einems Antwort, Gelbard u Sarajevu, Tuđman primio Kleina.

Die ersten Unterschiede zwischen den analysierten deutschen und österreichischen Nachrichtensendungen zeigen sich im Sprach- bzw. Sprechverhalten der Redakteure. Die deutschen Redakteure verwenden nämlich auch in der Meldung selbst elliptische Konstruktionen, die in den analysierten Sendungen von ihren österreichischen Kollegen nicht verwendet werden - z.B. Gazelle statt Reh, Angeklagt unter anderen X.Y. Ein besonderes Merkmal des Sprachverhaltens österreichischer Redakteure sind dagegen komplizierte Satzstrukturen mit Nebensätzen ersten und zweiten Grades, vor allem in Gesprächen mit ihren Gästen im Studio. Der Wortschatz in den analysierten 
deutschen Nachrichtensendungen entspricht dem binnendeutschen Standard. In den analysierten österreichischen Nachrichtensendungen werden fast ausnahmslos österreichisch markierte Lexeme verwendet, z.B. Spital, Spitalsreform, Meldungsüberblick, Betriebsobmann. In der phonetischen Realisation besteht ein klarer Unterschied in der Artikulation und in der Intonation der deutschen Redakteure, die eine dialektal gefärbte Aussprache vermeiden, und der österreichischspezifischen Aussprache ihrer Kollegen im ZIB2, bei einigen Sprechern vorwiegend wienerischer Prägung,

Die analysierten kroatischen Nachrichtensendungen unterscheiden sich von den deutschen und österreichischen durch die Produktions- bzw. Entscheidungsprozesse der Nachrichten. Deshalb rückt der Schwerpunkt der Analyse auf die spezifischen politischen Restriktionen der Nachrichtenerzeugung und ihrer (manipulativen) Verwendung. Das, woran die Redaktionspolitik des kroatischen Fernsehens erkannt wird, ist die Personalisierung der Nachricht. An erster Stelle stehen immer Informationen aus Kroatien, unabhängig von der Bedeutung ausländischer Nachrichten. So wurde beispielsweise am 1. Mai 1996 zunächst über eine lokale Feier in Zagreb berichtet und erst dann über das historische Treffen Arafat - Rabin.

Ein Merkmal des phonetischen Verhaltens kroatischer Redakteure und Nachrichtensprecher ist die strikte Befolgung der standardisierten Aussprache, ohne dialektale Färbung. Ein Vergleich mit den deutschsprachigen Sendungen auf der Ebene der Realisation syntaktischer Strukturen ist wegen unterschiedlicher Sprachstruktur nicht sinnvoll.

Der Kommentar gehört nicht zur Grundstruktur der Nachrichtensendung. Kommentare werden nur gelegentlich gesendet - der Kommentar sollte nämlich, im Unterschied zur informativen Qualität der Nachricht, analytische Qualität haben, wobei aber die Gefahr besteht, daß je nach dem Interessenstandpunkt und den Fähigkeiten des Kommentators das subjektive Element $\mathrm{zu}$ deutlich anzumerken ist, was das ungebrochene Vertrauen in die Information erheblich abschwächen könnte. Kommentare kommen sowohl in heute, im ZIB2 sowie in Dnevnik vor, mit dem Unterschied, daß die Kommentare im ZDF und Ö2 vor allem analytisch orientiert sind, wogegen die Funktion der Kommentare im kroatischen Fernsehen nichts anderes als die Rechtfertigung der offiziellen Staatspolitik der regierenden Partei ist.

Viel häufiger als Kommentare sind in der Tagesschau Korrespondentenberichte. Ein Korrespondentenbericht ist nach ähnlichen Prinzipien wie eine Meldung zugeschnitten; durch die Art seiner Aufmachung der Augenzeugenillusion leistet er dem Glauben Vorschub, das Ereignis selbst mitzuerleben. Das gemeinsame Merkmal des Sprechverhaltens der Korrespondenten in den analysierten Nachrichtensendungen ist das mundartlich gefärbte Sprechen.

Die Grundmerkmale der Textsorte Fernsehnachrichten sind die indirekte, einseitige Kommunikation sowie die streng schematisierte Einteilung der Nachrichtenblocks. Der wesentliche Inhalt von Nachrichten besteht in der Personalisierung politischer Ereignisse, die - vor allem im kroatischen Fernsehen - eher zu einer 
Werbung für die Personen wird, die genannt werden, als zu einer politischen Information. Dies führt zu einer Hierarchie zwischen Politikern, die in der Tagesschau auftreten dürfen und solchen, die es noch nicht geschafft haben. Die vielen, eigens fürs Fernsehen gestellten Berichte von Ereignissen, deren einzige Bedeutung eben nur gerade darin liegt, daß sie vom Fernsehen verarbeitet werden, und das Auftreten schon altbekannter Personen in ebenso bekannten Posen lassen die Welt wieder überschaubar werden. (B. Badura - K. Gloy, Soziologie der Kommunikation, Stuttgart-Bad Cannstatt 1972). So schaffen die Fernsehnachrichten eine Art Pseudoumgebung, vielleicht sogar Pseudowirklichkeit, die der Realität nicht standhält.

\section{Zusammenfassung}

Aufgrund der Kriterien der Merkmalhomogenität wird die Nachrichtensendung als Textsorte bestimmt. Ein Vergleich deutscher, österreichischer und kroatischer Sendungen zeigt, daß sie die gleiche Grundstruktur aufweisen. Ein Merkmal kroatischer Nachrichten ist die Personalisierung politischer Ereignisse.

Povzetek

BESEDILNA VRSTA 'TELEVIZIJSKA POROČILA' - KONTRASTIVNO

Besedilna vrsta 'televizijska poročila' je opredeljena na podlagi homogenosti njenih značilnih elementov. Primerjava tovrstnih nemških, avstrijskih in hrvaških oddaj pokaže enako osnovno strukturo. Ena od značilnosti hrvaških poročil je personaliziranje političnih dogodkov. 